\title{
Osteogenesis Imperfecta or Fanconi-Bickel Syndrome? (Report of a Very Rare Disease Due to New Mutation on GLUT2 Gene)
}

\section{A R T I C L E I N F O}

Article Type

Case Report

\section{Authors}

Shafeghati Y.* $M D$

Sarkheil P. ${ }^{1} M D$,

Baghdadi T. ${ }^{2} M D$

Hadipour F. ${ }^{3} M D$

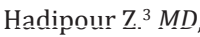

Noruzinia M. ${ }^{4} M D, P h D$

\section{How to cite this article}

Shafeghati Y, Sarkheil P, Baghda-

di T, Hadipour F, Hadipour Z, No-

ruzinia M. Osteogenesis Imperf-

ecta or Fanconi-Bickel Syndro-

me? (Report of a Very Rare Di-

sease Due to New Mutation on

GLUT2 Gene). Sarem Journal of

Reproductive Medicine. 2017;1(2):

73-76.

\section{A B S T R A C T}

Introduction Fanconi-Bickel disease is a rare disorder in the metabolism of carbohydrates. The disease is transmitted by heredity recessive autosomi. Somebodies have classified this diease as a glycogenosis that does not appear to be correct, because the cause of this disease not a disorder in the metabolism of glycogen, realy it is due to impairment of saccarides transfer into the membranous GLUT2 channel. The properties consist of: hepatorenal glycogenose, proximal RTA, impaired glucose and galactose consumption, manage resistant hypophosphatemic rickets and delay the growth resulting from it. But being non-typical cues could wrongly lead to another initial diagnosis like osteogenesis imperfecta.

Patient Information 3.5-year-old girl with the probable diagnosis of osteogenesis imperfecta was admitted for periodical treatment by pamydronat. The child resulting from consanguineous marriages and in history of pregnancy and maternity there was not any problem. Because of the delay in motion and repeated fractures, suspicion to osteogenesis imperfecta disease was initially raised. In the follow up of the next turned the patient revealed resistant rickets. Gradually hepatomegaly appearead and the biopsy emphasized glycogen accumulation. Then gradually renal affliction -fanconi syndrome and RTA- were revealed.

Conclusion According to the mentioned symptoms, the very rare diseases Fanconi-Bickel syndrome was raised for the patient. By molecular Study on operating gene called Glucose transporter 2 (GLUT2), this disease was confirmed. This type of mutant is recognized for the first time that has not already been reported in medical resources.

Keywords Fanconi-Bickel Syndrome; GLUT2 gene; New Mutation; Osteogenesis Imperfecta
*"Sarem Fertility \& Infertility Research Center (SAFIR)" and "Sarem Cell Research Center (SCRC)”, Sarem Hospital, Tehran, Iran

${ }^{1}$ Genetics Department, Sarem Women's Hospital, Tehran, Iran

${ }^{2}$ Imam Khomeini Hospital, Tehran University of Medical Sciences, Tehran, Iran

3"Sarem Fertility \& Infertility Research Center (SAFIR)", "Genetics Department" and "Sarem Cell Research Center (SCRC)", Sarem Women's Hospital, Tehran, Iran

${ }^{4}$ Medical Genetics Department, Faculty of Medical Sciences, Tarbiat Modares University, Tehran, Iran

\section{Correspondence}

Address: -

Phone: -

Fax: -

dr.yshafagh@gmail.com

\section{Article History}

Received: January 11, 2016

Accepted: May 18,2016

ePublished: June 15, 2017

\section{CIT A T I O N L I N KS}

[1] Fanconi-Bickel syndrome-the original patient and his natural history, historical steps leading to the primary defect, and a review of the literature [2] Fanconi-Bickel syndrome-a congenital defect of facilitative glucose transport [3] Fanconi-Bickel syndrome [4] Diabeteslike renal glomerular disease in Fanconi-Bickel syndrome [5] Hepato-renal glycogenosis with complex tubulopathy. 2. Cases of a new entity [6] Sequence, tissue distribution, and chromosomal localization of mRNA encoding a human glucose transporterlike protein [7] Organization of the human GLUT2 (pancreatic beta-cell and hepatocyte) glucose transporter gene [8] Mutation analysis of the GLUT2 gene in patients with Fanconi-Bickel syndrome [9] The mutation spectrum of the facilitative glucose transporter gene SLC2A2 (GLUT2) in patients with Fanconi-Bickel syndrome 


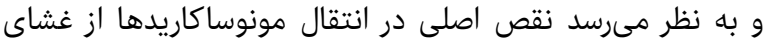

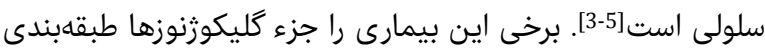

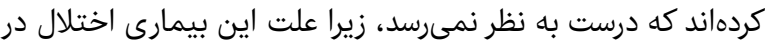

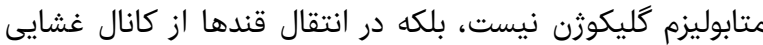

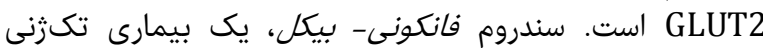

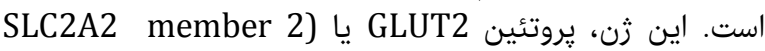
اجزاي خاد (facilitated glucose transporter اجزاى خانواده بزرى يروتئينى است و مسئول انتقال قندها از از

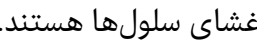

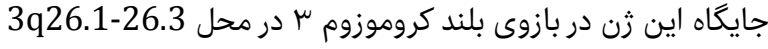

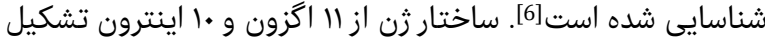

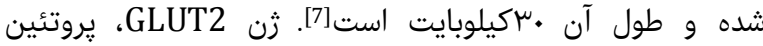

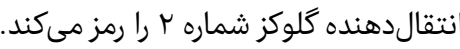

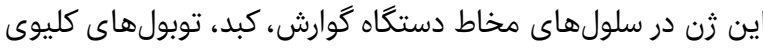

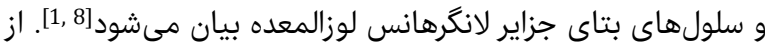

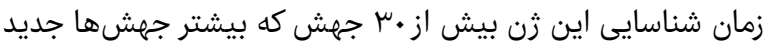

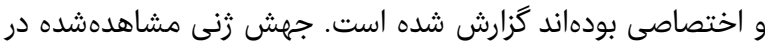

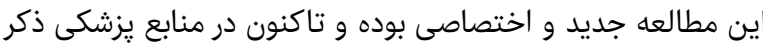

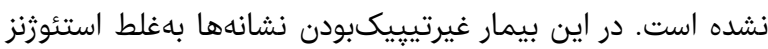

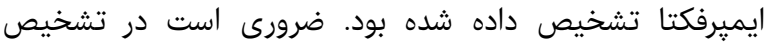
افتراقى، راشيتيسمهاى مقاوم به درمان اين بيمارى هم دردي در فهرست

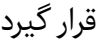

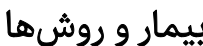

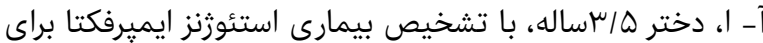

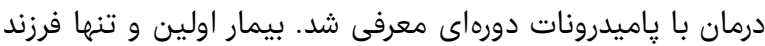

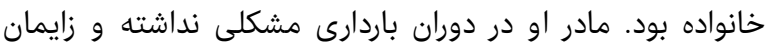

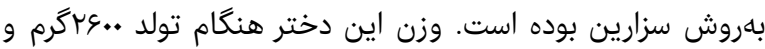

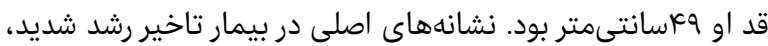

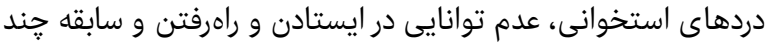

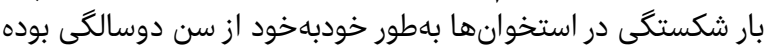

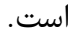

با تشخيص بيمارى استئوزنز، اولين دوز ياميدرونات به بيمار تزريق

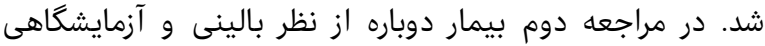

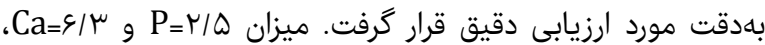

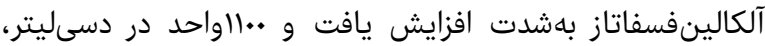

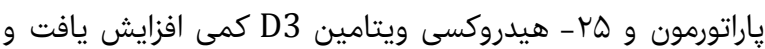

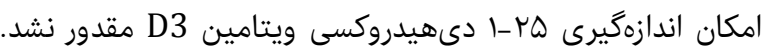

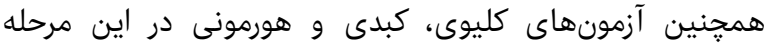

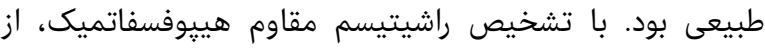

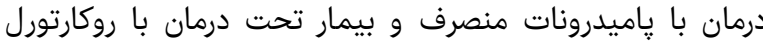

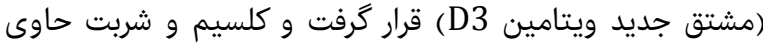

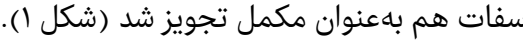

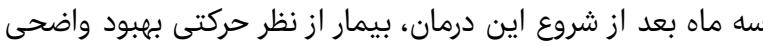

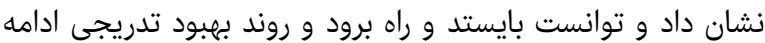

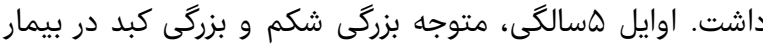

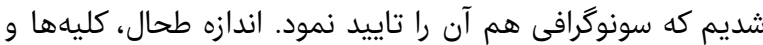

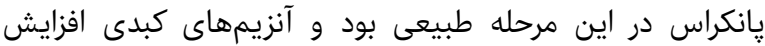

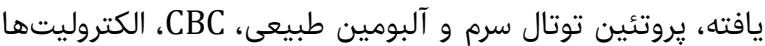

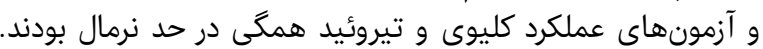

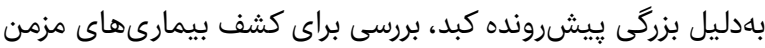

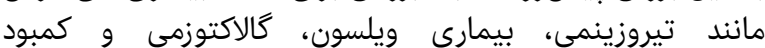

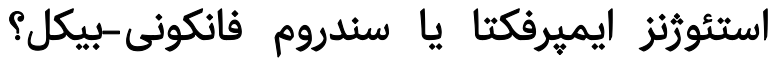

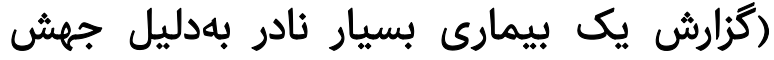

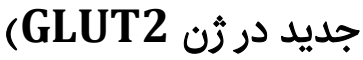

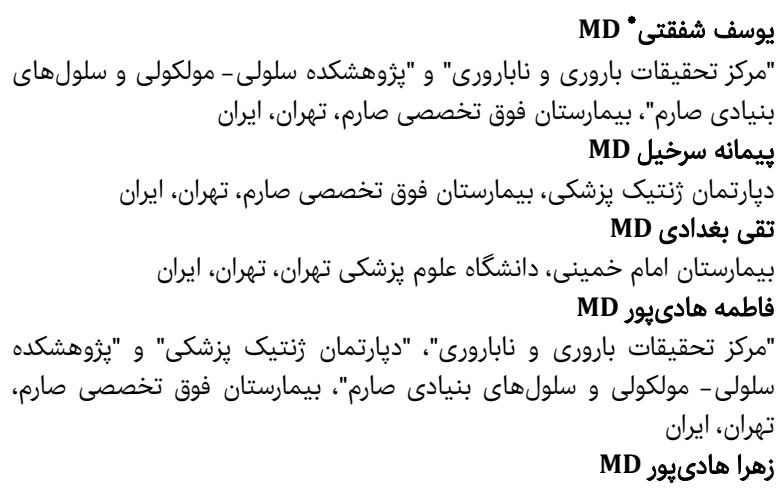

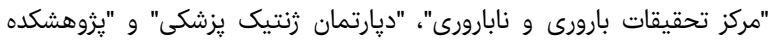

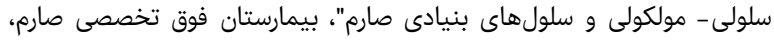

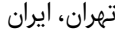

MD, PhD مهرداد نوروزىنيك انيكيان

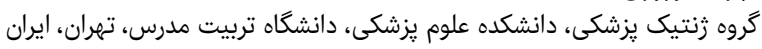

جكيده

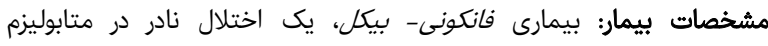

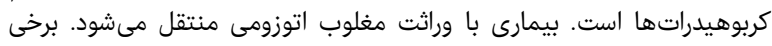

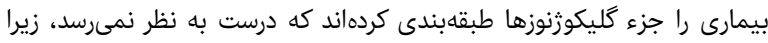

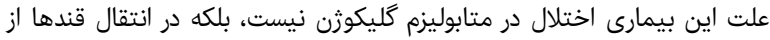

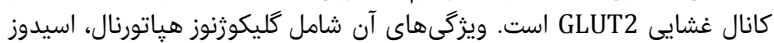

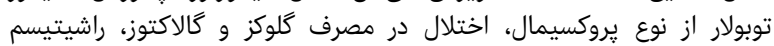

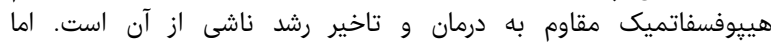

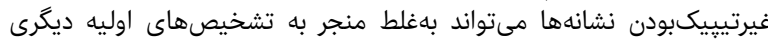

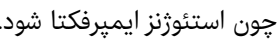

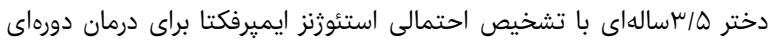

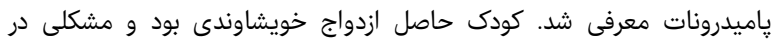

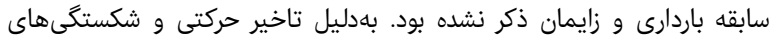

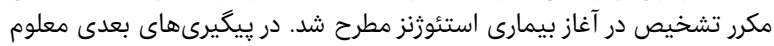

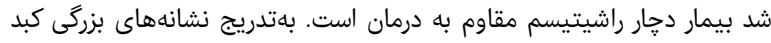

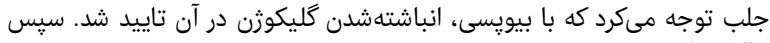

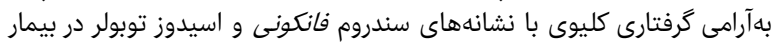

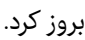

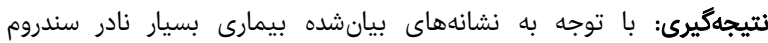

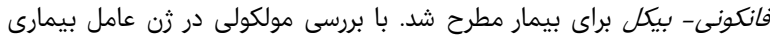

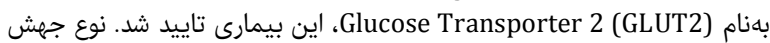
در بيمار، براى اولينبار شناسايى شد كه يِيش از اين در منابع يزشكى تزارش نشده بود.

كليدوازهها: سندروم فانكونى بيكل، ثن GLUT2، جهش جديد، استئوثنز ايميرفكتا

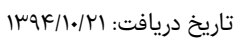

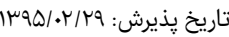

"نويسنده مسئول: dr.yshafagh@gmail.com

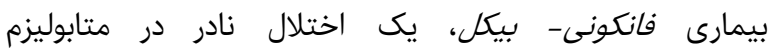

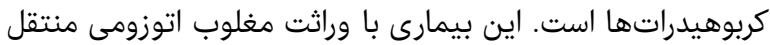

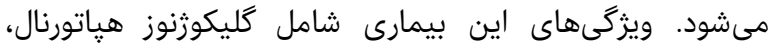

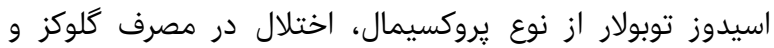

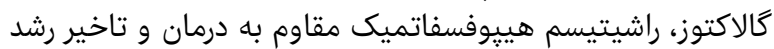

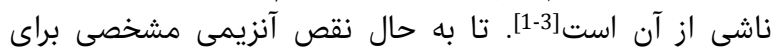

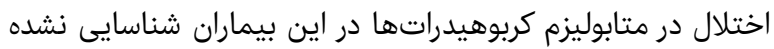




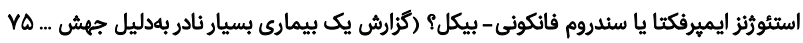

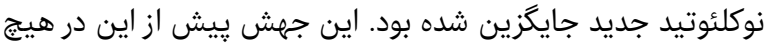

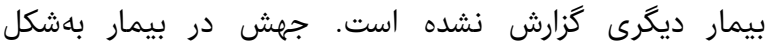

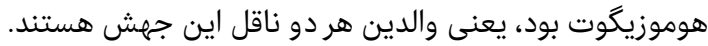

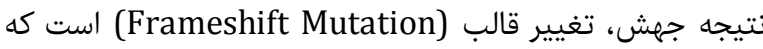
سبب توليد يروتئين كوتاهترى با مشخصات زير زير است: c.685_701del GCCATCCTTCAGTCTCT ins كه بدون ترديد جهشى بيمارىزا CAGAAAA; p.A229 QfsX19

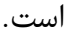

بحث

مشخصات فانكونى- بيكل از تركيب راشيتيسم هييوفسفاتميك

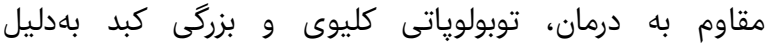
انباشتهشدن كليكوثن در هياتوسيتها استان.

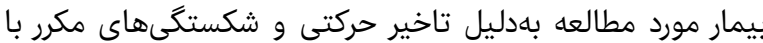

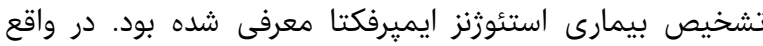

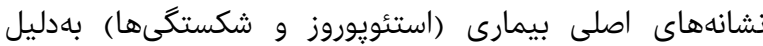

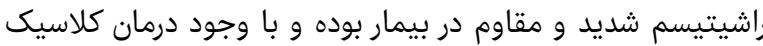

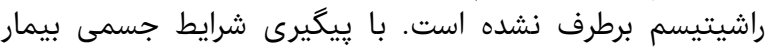

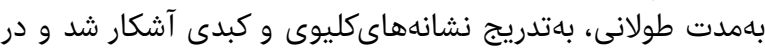

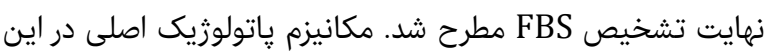

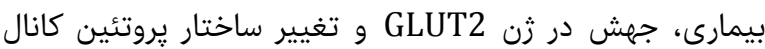

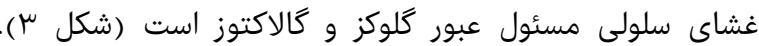

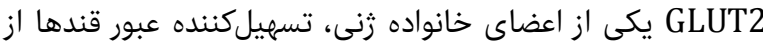
غشاى سلولى است (شكل سام).

\section{Control of Insulin Secretion}

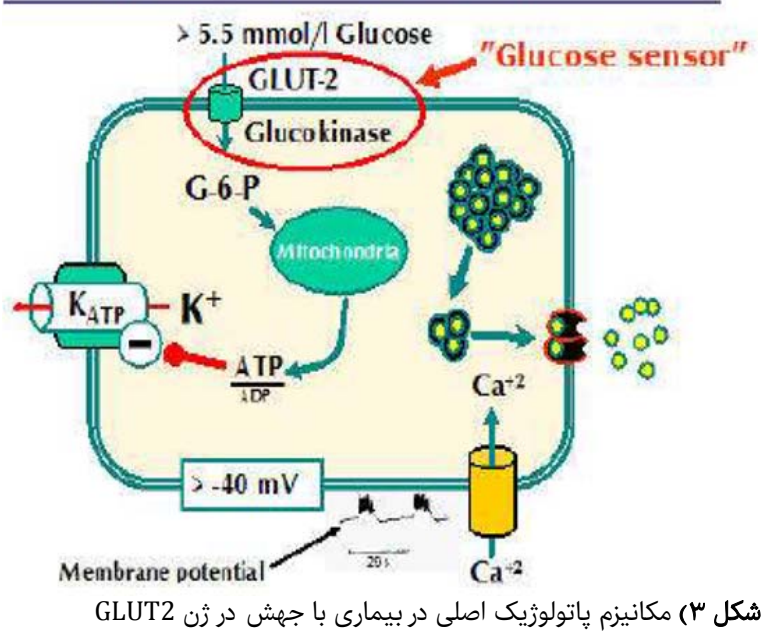

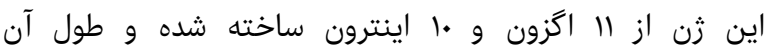

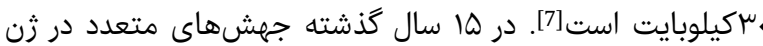

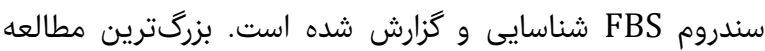

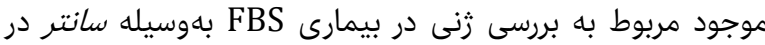

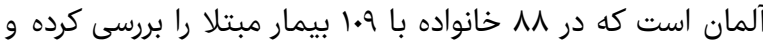

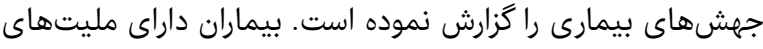

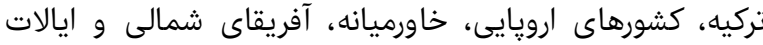

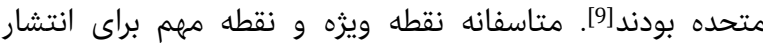

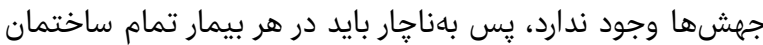

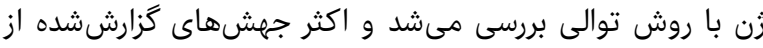

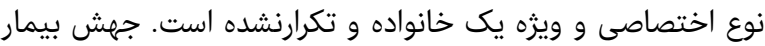

آلفا-ا-آنتىترييسين انجام شد كه همگى در حدود طبيعى بود و وخدار

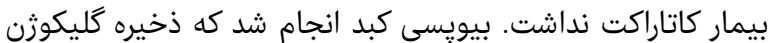

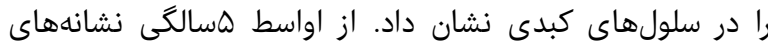

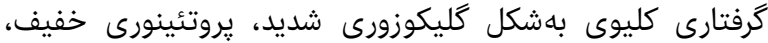

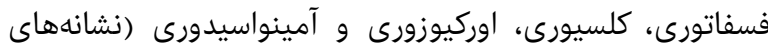

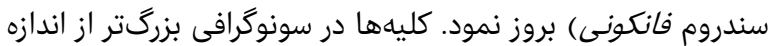

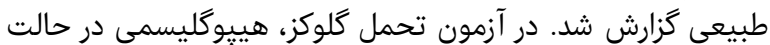

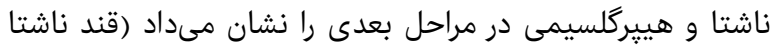

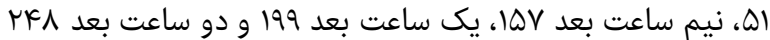

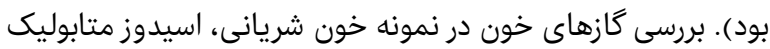

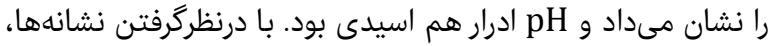

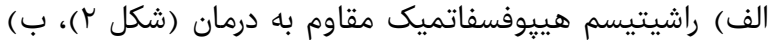

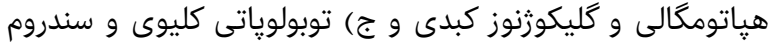

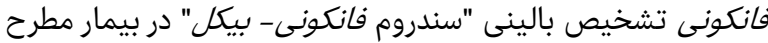

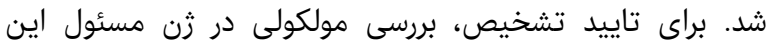

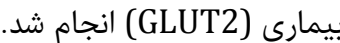

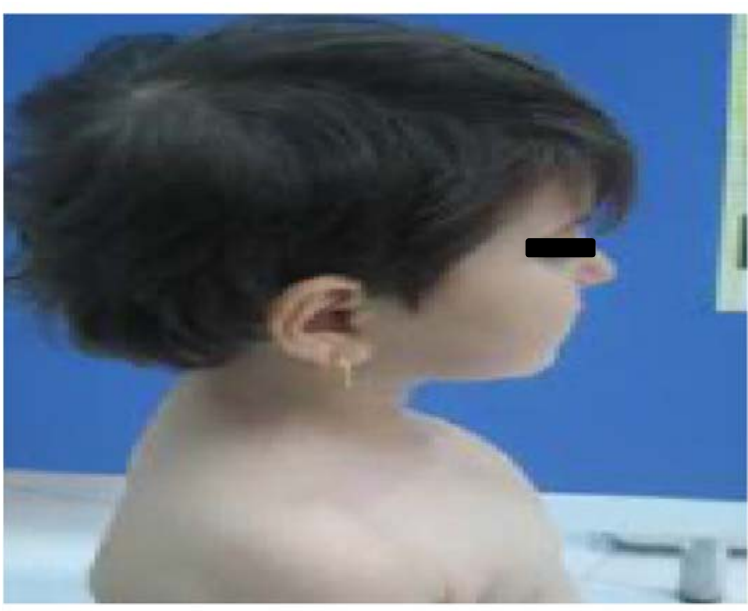

شكل () تصوير بيمار مورد بررسى در آغاز درمان

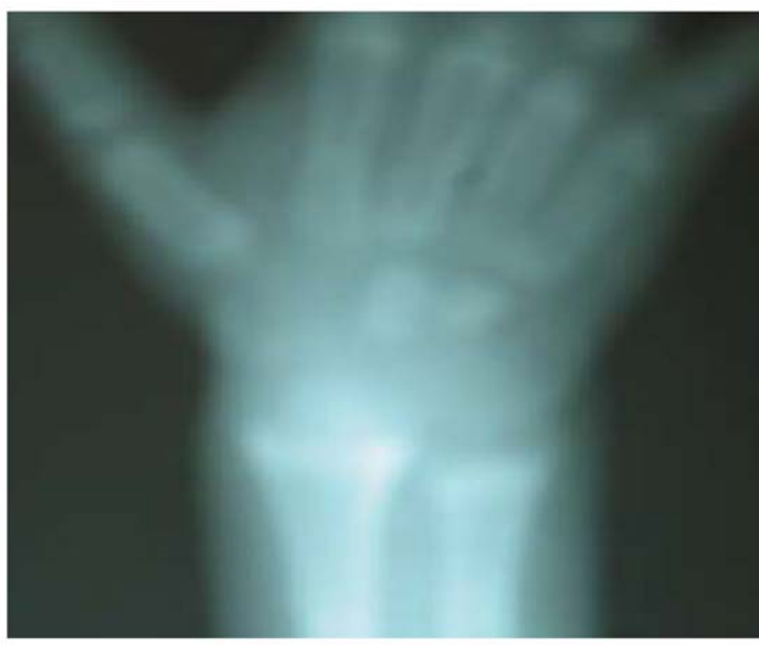

شكل r) عكس راديولوزى بيانكر راشيتيسم

با تشخيص بالينى سندروم فانكونى- بيكل، تمام اخزونها،

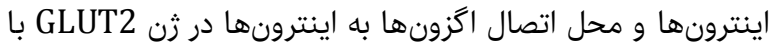

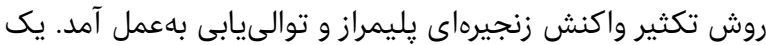

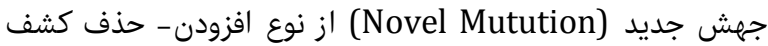

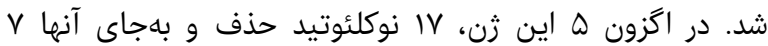

Volume2, Issue1, Spring 2017 
تشكر و قدردانى: نويسندگان از خانواده بيمار بهدليل همكارى

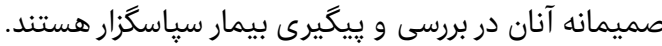

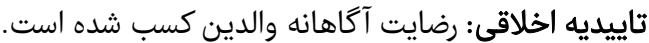

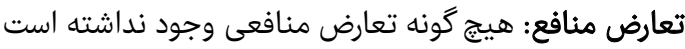

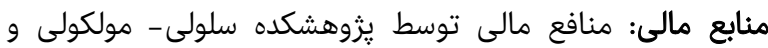
سلولهاى بنيادى صارم تامين شده مالع است توسط

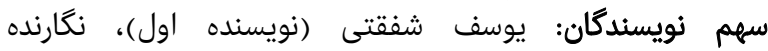

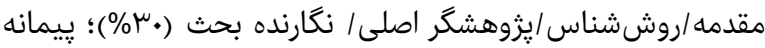

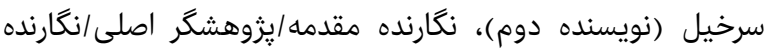

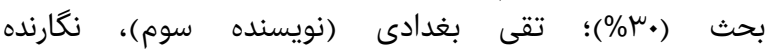

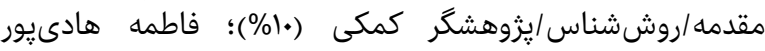

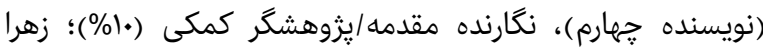

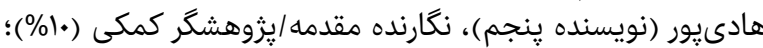

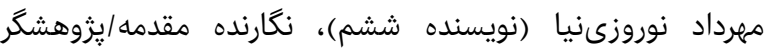
كمكى (\%)

منابع

1- Santer R, Schneppenheim R, Suter D, Schaub J, Steinmann B. Fanconi-Bickel syndrome--the original patient and his natural history, historical steps leading to the primary defect, and a review of the literature. Eur J Pediatr. 1998;157(10):783-97.

2- Santer R, Steinmann B, Schaub J. Fanconi-Bickel syndrome--a congenital defect of facilitative glucose transport. Curr Mol Med. 2002;2(2):213-27.

3- Manz F, Bickel H, Brodehl J, Feist D, Gellissen K, Gescholl- Bauer B, et al. Fanconi-Bickel syndrome. Pediatr Nephrol. 1987;1(3):509-18.

4- Berry GT, Baker L, Kaplan FS, Witzleben CL. Diabeteslike renal glomerular disease in Fanconi-Bickel syndrome. Pediatr Nephrol. 1995;9(3):287-91.

5- Odievre M. Hepato-renal glycogenosis with complex tubulopathy. 2. Cases of a new entity. Rev Int Hepatol. 1966;16(1):1-70. [French]

6- Fukumoto H, Seino S, Imura H, Seino Y, Eddy RL, Fukushima $Y$, et al. Sequence, tissue distribution, and chromosomal localization of mRNA encoding a human glucose transporterlike protein. Proc Natl Acad Sci U S A. 1988; 85(15):5434-8.

7- Takeda J, Kayano T, Fukomoto H, Bell GI. Organization of the human GLUT2 (pancreatic beta-cell and hepatocyte) glucose transporter gene. Diabetes. 1993;42(5):773-7.

8- Sakamoto 0, Ogawa E, Ohura T, Igarashi Y, Matsubara Y, Narisawa K, et al. Mutation analysis of the GLUT2 gene in patients with Fanconi-Bickel syndrome. Pediatric research. 2000;48(5):586-9.

9- Santer R, Groth S, Kinner M, Dombrowski A, Berry GT, Brodehl J, et al. The mutation spectrum of the facilitative glucose transporter gene SLC2A2 (GLUT2) in patients with Fanconi-Bickel syndrome. Hum Genet. 2002;110(1):21-9.

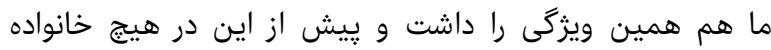

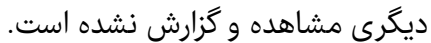

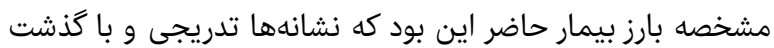

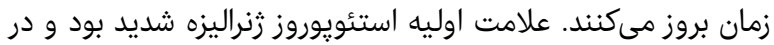

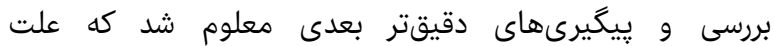

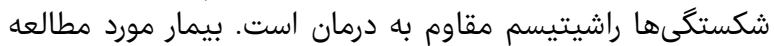

در حال حاضر |l سال دارد (شكل \& א).

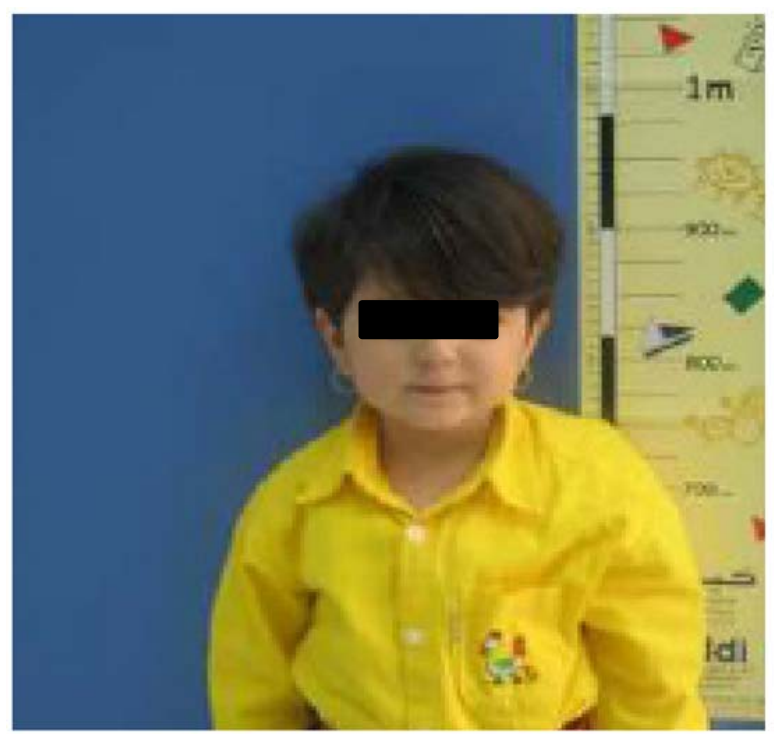

شكل \&) تصوير بيمار در ادامه درمان تا كنون در سن السالكى

بعد از تشخيص قطعى، بيمار تحت درمان با نشاسته ذرت،

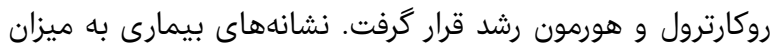

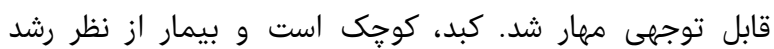

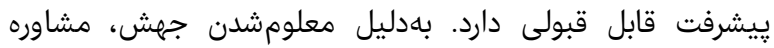

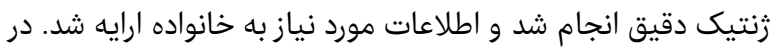

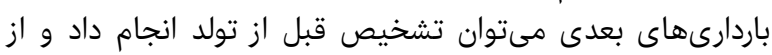

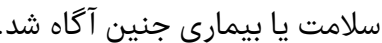

سندروم فانكونى- بيكل را بايد در تشخيص افتراقى علل

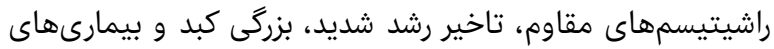

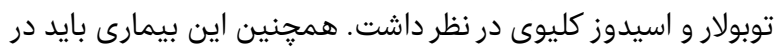

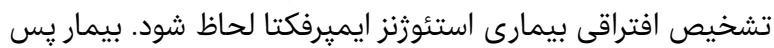

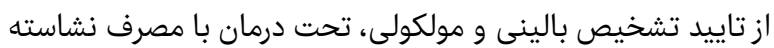

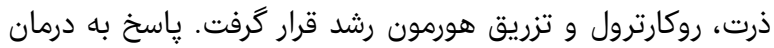

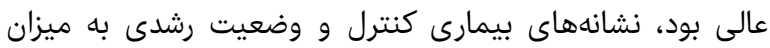

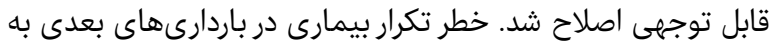

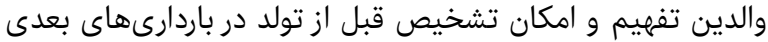

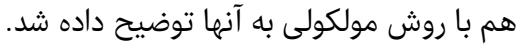

